Thorax (1969), 24, 391.

\title{
Transplantation of the heart and both lungs II. Experimental cardiopulmonary transplantation
}

\author{
D. B. LONGMORE ${ }^{1}$, D. K. C. COOPER ${ }^{2}, R$. W. HAL L L \\ J. SEKABUNGA ${ }^{4}$, A N D W. WE L C H ${ }^{5}$ \\ From the Departments of Surgery and Anaesthetics, National Heart Hospital, London
}

\begin{abstract}
It is estimated that an unknown, yet possibly large, number of patients would benefit from transplantation of the heart and both lungs if technically, physiologically, and immunologically feasible. In this paper we attempt to explore the main non-immunological areas in which we feel that cardiopulmonary transplantation requires further evaluation. A technique is described by which the heart and lungs, as one unit, can be removed from a donor animal, and viability of these organs can be maintained for several hours by autoperfusion (circulation being through the coronary and pulmonary vessels) with positive pressure ventilation via the trachea. This simple heart-lung preparation preserves the organs concerned for sufficient time to allow preparation of the recipient, transport of the donor organs, and tissue typing to be carried out. Our technique of implanting these donor organs into the recipient is also described. We have carried out this operation on approximately $100 \mathrm{dogs}$ and have been impressed by the good cardiac function obtained, but spontaneous respiratory function has been either absent or inadequate to sustain life for more than a few hours. It would appear that dogs cannot tolerate bilateral pulmonary denervation, and our findings are discussed in the light of other work on this subject. Work on primates suggests that man would be able to undergo this procedure successfully. The organizational and ethical problems involved in cardiac and cardiopulmonary transplantation are briefly discussed.
\end{abstract}

The Registrar General's (1967) Statistical Review of England and Wales for the year 1965 lists 152,718 deaths from arteriosclerotic and degenerative heart disease and 6,216 from chronic rheumatic heart disease. Many of the former group of patients might be very old with generalized arteriosclerosis and would not be considered for heart transplantation, but there remains a large number of middle-aged subjects who would be suitable for any possible form of treatment. Although the day is foreseen when these disorders may be prevented or satisfactorily treated by medical therapy, it is likely that, with the present rapid expansion of knowledge of tissue typing and immuno-suppression, heart transplantation will be a means of treatment in the intervening years.

Many of these patients have secondary lung damage and would benefit from transplantation of the heart and one or both lungs. In fact, in a

\footnotetext{
'Consultant Physiologist

${ }^{2}$ British Heart Foundation Surgical Research Fellow

'Senior Registrar in Anaesthetics

'Rockefeller Fellow

${ }^{5}$ British Council Surgical Research Fellow
}

certain percentage of such patients, this procedure, if technically and physiologically feasible, would be the only possible means of treatment. Children who are unlikely to reach maturity because of complex intrathoracic congenital defects would also benefit, but the problems of growth will have to be overcome.

Heart-lung transplantation may also have a part to play in the treatment of various lung diseases which affect the function of the heart, notably chronic bronchitis and emphysema, and it is conceivable that certain advanced cases of carcinoma of the bronchus involving mediastinal tissues may be treatable only by total excision of the heart and both lungs with homograft replacement. In 1965 there were 26,042 deaths from chronic bronchitis (with and without emphysema) and a further 18,300 from primary neoplasms of the bronchus, trachea, and lung.

The biggest single problem in the treatment of these patients will eventually lie in the provision of sufficient donor organs and in their satisfactory preservation. The source of such donor organs will be largely from patients dying from head 
injuries and neoplasms of the central nervous system. In 1965 there were 9,902 deaths from the former cause and 1,780 deaths from the latter. This would provide only 11,682 possible donors to supply a very much greater potential recipient population. The answer to this problem of supply and demand may prove to lie ultimately in the use of heterografts from anatomically and immunologically suitable animals or in the development of artificial mechanical organs, though the difficulties presented by koth of these possibilities are enormous.

Homotransplantation of the heart and both lungs would seem to have a place in an as yet unknown but definite number of patients with heart and lung disease. Such a procedure incorporates some of the problems of the individual transplantation of the heart or lung. Homotransplantation of these organs individually has been studied extensively by many workers. Shumway, Lower, and Stofer (1966) and Cooper (1968) have recently reviewed the subject of homotransplantation of the heart, and Hardy and Alican (1966) have reviewed the problems associated with lung transplantation.

Studies of transplantation of the heart together with both lungs as a functioning unit are few, and many questions remain to be answered, in particular, the effect on respiration of denervation of both lungs (Cooper, 1969).

Six major problems must be considered and overcome in the transplantation of any vital organ :

1. The technical problems of the actual transplantation operation ;

2. The physiological problems involved in totally detaching an organ from its surrounding tissues and, in particular, from dividing its nerve supply and reimplanting it in another animal ;

3. The immunological problems of rejection of the 'foreign' organ ;

4. The problems of preserving the viability of the donor organ while it remains exteriorized from either donor or recipient ;

5. The problems of preserving the viability of the recipient after its own diseased organ has been excised and before the donated organ has successfully resumed its special function;

6. The ethical and organizational problems involved in providing an adequate donor supply. These are highlighted in heart and heart-lung transplantation.

We have concerned ourselves with problems 1 , $2,4,5$, and 6 in regard to transplantation of the heart and both lungs. In this paper we describe our experiences with a simple method of short $\frac{\vec{*}}{\stackrel{3}{*}}$ term preservation of the heart and lungs, enabling transport of these organs from one centre to an- $\frac{E}{0}$ other, and allowing time for tissue typing. This $\overline{\bar{n}}$. suggested method would be equally valuable as $\overline{\mathrm{D}}$ a means of short-term storage and transport, whether the heart and both lungs or either of ${ }^{\infty}$ these organs were to be used. We also describe $\overrightarrow{0}$ an operative technique for transplantation of the $\overrightarrow{\vec{H}}$ heart and both lungs, and briefly present some ${ }_{\sigma}^{\omega}$ of our observations regarding the physiology of the transplanted organs. Our recipient animals have not survived long enough for us to study the immunological problems involved. The reci- $-\vec{\omega}$ pient is maintained on a pump-oxygenator while ${ }^{\omega}$ the transplantation procedure is being carried out, 0 but at the present time there is no long-term? supportive method available to the patient with severe heart disease. Such mechanical support would greatly facilitate the treatment of a patient $\overrightarrow{0}$ about to undergo, or recovering from, hearto transplantation. The ethical and organizational problems involved in such transplantation areo discussed briefly.

\section{EXPERIMENTAL TECHNIQUES}

The experimental procedures to be described have been carried out in dogs, generally mongrels, weigh ing from 10 to 35 kilograms. Efforts were made to perform the operation on two dogs well matched in? size and weight. No attempt was made to cross match the blood of the two animals.

TECHNIQUE OF PREPARATION AND SHORT-TERM PRE SERVATION OF THE DONOR HEART AND LUNGS The donor animal is anaesthetized with pentobarbitone sodium (Nembutal), $25 \mathrm{mg}$./ kg. body weight, and maintained on a mechanical ventilator on $100 \%$ oxygen. The chest is opened by a bilateral transverse thoracotomy in the 4th intercostal space. The azygo o vein is doubly ligated and divided near its junction with the superior vena cava. The superior and inferior venae cavae are mobilized but not yet ligated. The trachea is dissected out, as are the brachiocephalico and left subclavian arteries. The arch and proxima descending aorta are mobilized, and the lungs areo freed by division of their pulmonary ligaments. The heart and lungs are dissected away from the deeper mediastinal structures, the dissection being carrieds out in the relatively avascular plane anterior to the oesophagus. The pericardium is excised.

At this stage of the procedure the donor anima is heparinized and is given $10 \mathrm{mg}$. dipytridamole intravenously, a drug which we have found valuable in dilating the coronary arteries and thus improvingo

${ }^{6}$ Persantin-Boehringer Ingeilheim Ltd. 
cardiac action. In earlier studies adrenaline was used with a similar effect, though this led to troublesome disorders of cardiac rhythm.

With the heart and lungs freed from the underlying structures, only the major vessels and trachea need to be divided to complete the dissection. First, the brachiocephalic and left subclavian arteries and superior vena cava are each doubly ligated and divided, followed immediately by ligation and division of the inferior vena cava (Fig. 1). The timing of these final procedures is crucial to the continuing function of the donor heart. If the atria are allowed to overdistend with the venous return from the inferior vena cava, following occlusion of the aorta, pulmonary oedema may ensue. If the inferior vena cava is ligated too soon, then the heart-lung preparation will not contain an adequate circulating volume and will be unable to generate sufficient pressure to sustain itself. Accordingly, during the removal it is necessary to watch the right atrium carefully or to measure atrial pressure. The trachea is divided as high as possible and intubated with an endotracheal tube which is tied in position. The donor lungs are gently inflated with oxygen, which can be carried out simply by placing a $\mathrm{T}$-piece in the line between the endotracheal tube and a small oxygen cylinder. If the open limb of the $T$-piece is occluded intermittently a simple form of positive pressure ventilation is initiated.

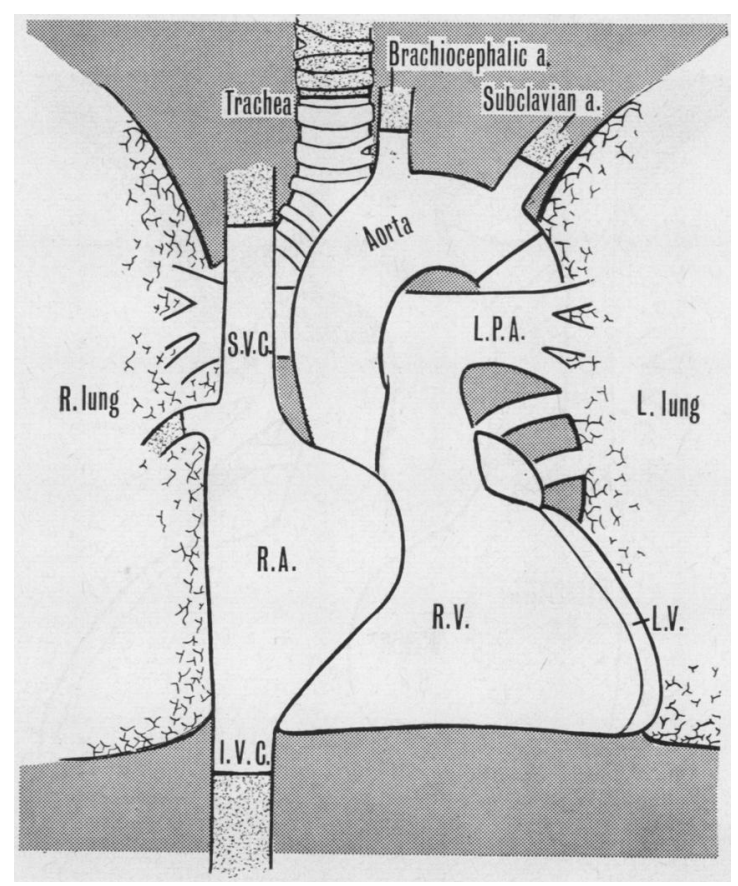

FIG. 1. Tissues (in white) removed from the donor animal.
The heart-lung preparation is now functioning as an independent unit and can be lifted from the donor animal. Attention is paid to oozing from small vessels in the preparation, for if the organs are to be transported and preserved for a few hours a considerable blood loss can result during this period. In the dissection of this specimen no attempt is made to preserve the distal bronchial arteries.

The donor heart and lungs or heart alone can be transplanted into the recipient animal immediately, or the heart-lung preparation can be preserved for several hours before the organs are inserted. Demikhov (1950) was the first to realize the potential of this preparation as a means of transporting the organs involved. Its great advantage as a means of temporary storage is its simplicity. As long as the lungs are kept gently oxygenated, the left heart will receive oxygenated blood with which it will supply the myocardium via the dilated coronary arteries. The desaturated blood will return via the coronary sinus to the right heart, and will be returned to the lungs for gaseous exchange.

During early studies the preparation was hung from a frame, but it was found that cooling and drying of the surface of the heart led to disturbances and eventual cessation of function within one to two hours. Subsequently, the specimen has been placed in either a bowl or plastic bag containing peritoneal dialysing solution with potassium added to $5 \mathrm{mEq} /$ litre. It is important to maintain the temperature of this fluid as near to $37^{\circ} \mathrm{C}$. as possible, for lowering the temperature leads to ventricular fibrillation. Immersion in such a warm solution has increased the survival time of specimens to at least six hours without difficulty.

In some animals we have simulated donor death with asphyxia, and in others cardiac arrest has occurred on induction of anaesthesia. In these animals the removal of the heart-lung preparation has been as described above, but in order to fill the arrested heart it is necessary to clamp the aorta, to elevate the hind limbs of the animal to improve the venous return, and to massage the heart. Removal of the specimen, its immersion in warm dialysing fluid, the administration of calcium, sodium bicarbonate, and adrenaline, and electrical defibrillation have on many occasions produced a heart-lung preparation which behaves in a similar manner to those preparations removed while still beating.

The preparation can therefore be transported and preserved in this basically physiological state for several hours by one attendant requiring nothing more than a small oxygen cylinder, some form of container for the fluid and organs, and some means of maintaining a temperature approximating to $37^{\circ} \mathrm{C}$.

In our opinion, the advantages of the above method of short-term storage are that it does not require the rather more elaborate facilities of hypothermia, artificial perfusion or hyperbaric 
oxygen. Major surgery such as heart substitution takes time to organize. It is advantageous to be able to obtain and store hearts in a simple manner which might be undertaken easily at peripheral hospitals where there may not be sophisticated facilities. It is also necessary to be able to transport the specimen readily.

Robicsek, Sanger, and Taylor (1963) have described a similar method of autoperfusion of the heart-lung preparation, but they incorporated a pressure-stabilizing 'buffer' bag of blood which was connected to the aortic stump and had to be hung up one metre above the preparation. The metabolic changes in the organs using this method of storage have been studied by Yamada, Bosher, and Richardson (1965). Our own studies will be reported fully later.

TECHNIQUE OF PREPARATION OF THE RECIPIENT ANIMAL AND IMPLANTATION OF THE DONOR HEART AND LUNGS Anaesthesia of the recipient animal is identical to that of the donor. The animal is placed in the left lateral position and the chest is opened in the right 5 th intercostal space extending through the sternum. After preliminary dissection, which includes careful preservation of both phrenic nerves, the venae cavae are separately cannulated, as is the left femoral artery, and normothermic cardiopulmonary bypass is established using a Proctor oxygenator with a clear fluid prime.

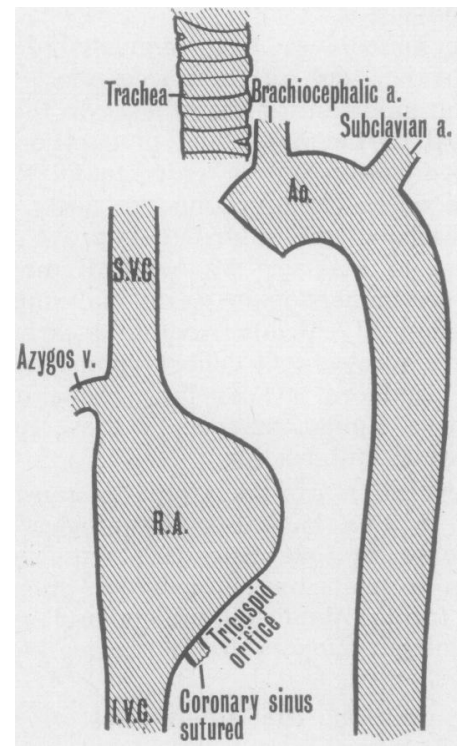

FIG. 2. Tissues (shaded) which remain in the recipient after excision of the helart and lungs.
Each lung is separately mobilized and excised, as are the ventricles, leaving in the recipient the entire? right atrium and the posterior wall of the left atriume (Fig. 2). The vagus nerves are disturbed as little as음 possible. The trachea is divided just above the carina. $\frac{\bar{\Phi}}{\bar{\alpha}}$ The coronary sinus opening into the right atrium is closed.

The donor specimen is then removed from its con-s tainer and placed in the left chest of the recipient, $\overrightarrow{0}$ and the right lung is passed behind the right atriumto lie in the right pleural cavity. The donor tracheac is passed through by the same route and reintubated with a sterile tube, and ventilation is re-established. $\vec{x}$ The two aortae are then anastomosed using an everting suture. An air needle is inserted and the clamp on the recipient aorta is released, driving air out of the junctional region. The clamp on the donor aorta is released, allowing coronary perfusion from theo pump-oxygenator. Ventilation of the lungs can then_ be discontinued. It is important to vent the donore right atrium quickly at this stage to prevent overdistension of the heart if ejection is not satisfactory.A linear incision is made in the donor right atriumb and this chamber is anastomosed to the host tricuspide ring. The tracheal edges are tailored and anastomosed (Fig. 3). Ventilation is re-established through an oral endotracheal tube and cardiopulmonary bypass is discontinued. The procedure requires 30-90 minutes bypass time. Two pleural drains are inserted and, if cardiac function is satisfactory and haemostasis has $\varrho$ been obtained, the chest is closed.

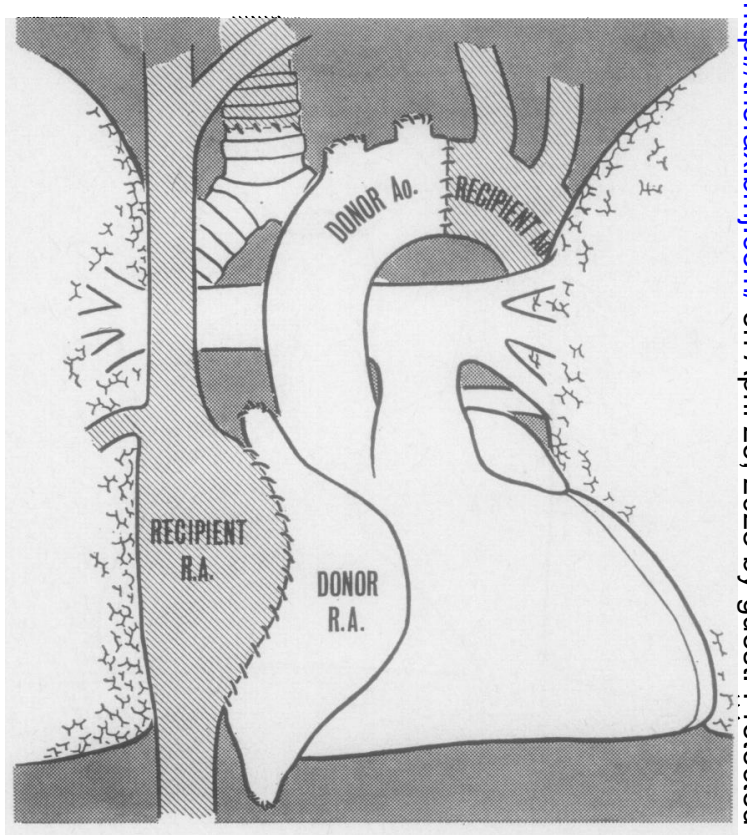

FIG. 3. The completed operation, after anastomosis of donor tissues (white) to the recipient tissues (shaded). 
The difficulties with this procedure in the dog are considerable. Like many others before us we have found that dogs do not tolerate cardiopulmonary bypass well, and we have lost many animals from the effects of this procedure. However, the major technical difficulty without doubt is the aortic anastomosis. In the dog the aortic wall is frequently of a soft, friable consistency, which we have found difficult to anastomose satisfactorily. We have experimented with many types of suture line and have found the incorporation of a narrow strip of Teflon to be beneficial in obtaining haemostasis. If haemostasis cannot be obtained here, we have noted that this continuous 'surgical' bleeding, even if relatively slight, combines with the effects of pumpoxygenation on the blood to lead to 'medical' bleeding which we have never been able to correct. The atrial anastomoses can also be a source of blood loss in the heparinized dog, but in general they present far less of a problem. It is similarly not without difficulty to gain an airtight tracheal anastomosis.

\section{RESULTS}

Approximately 100 experiments have been carried out, the results of which can be divided into four main groups:

GROUP 1 In these animals, particularly in earlier experiments, due to technical problems the survival time was extremely short. No conclusions have been drawn from this group.

GROUP 2 In these cases the donor heart was in a very poor condition at the outset of the procedure. This group contains those hearts which fibrillated during their removal from the donor animal and which could not be defibrillated. In these cases the organs were cooled in iced saline until implanted in the host, when they were rewarmed. Such hearts have uniformly done badly. Although definite pathology was present in some cases, the causes for this early fibrillation are not fully understood, but it has been noticeable that these donor animals were generally in extremely poor condition. We have not drawn conclusions here either.

GROUP 3 In the hours following the operation these animals were haemodynamically stable and satisfactory, but they continued to ooze. By replacing the blood loss in these dogs it has been possible to make observations regarding their cardiac and respiratory function.
This group contains a large number of dogs. Death occurred 6-9 hours post-operatively from hypovolaemia when it became impossible to replace the blood lost.

However, it is noteworthy that in these dogs cardiac function was good. Slow idio-ventricular rates were rare. The heart beat strongly and with a regular rhythm, maintaining an adequate systemic blood pressure and a variable pulse rate between 70 and 150 beats/minute. These facts, together with the observation that pulmonary oedema did not occur, suggest that left heart function was good. Central venous pressure was maintained within normal levels, confirming that right heart function was equally satisfactory.

The electrocardiogram usually showed some changes of myocardial damage while the heart was in the early post-operative phase, but there was a slow, progressive return towards normality.

Respiratory function was by no means as satisfactory as cardiac function. One-third of the animals in this group showed no return of spontaneous respiration and required maintenance by mechanical ventilation throughout the postoperative phase. The majority of the others regained only a gasping form of respiration which was totally inadequate to support life for more than a few minutes. This rapidly diminished to total apnoea and the animal would have died unless it had been ventilated artificially. A minority of dogs breathed more satisfactorily, but it was rarely adequate to maintain good oxygenation of the blood for more than one to two hours.

Many of these animals showed signs of central nervous system recovery following the procedure, with the return of pupillary, eyelid, and other reflexes and of spontaneous movements, which at times have been vigorous enough to require sedation. Many also passed urine.

Although complicated by the need for continuous fluid replacement, the general picture of this group was one of satisfactory cardiac function but inadequate respiratory function.

GROUP 4 A small number of dogs have been complete technical successes with good haemostasis, though the maximal survival, even in this group, has been only 25 hours. Our longest survivor illustrates the observations made from these animals. First, it is notable that the recipient and donor were litter-mates and were particularly healthy. They were only 18 months old. Their extremely good condition and youth were quite possibly important factors in their increased survival time. The recipient was perfused for only 
45 minutes. When the chest was closed the systemic blood pressure was $110 / 60 \mathrm{~mm}$. $\mathrm{Hg}$ (the pre-operative blood pressure of the recipient had been $220 / 150 \mathrm{~mm}$. $\mathrm{Hg}$ and of the donor $140 / 95$ $\mathrm{mm} . \mathrm{Hg}$ ) and the central venous pressure $2 \mathrm{~cm}$. $\mathrm{H}_{2} \mathrm{O}$. The dog began to make intermittent spontaneous respiratory movements 2 hours after closure of the chest and was 'fighting' the respirator 1 hour later, sufficiently so that artificial respiration was discontinued. At this time blood gases were satisfactory $(p \mathrm{H} 7 \cdot 4$, standard bicarbonate 19, $\mathrm{PCO}_{2} 27 \cdot 5, \mathrm{PO}_{2} 230$ ) and the animal bireathed spontaneously for the next $3 \frac{1}{2}$ hours without difficulty. The respirations were deep, slow, and regular, though not far removed from normality. After $1 \frac{1}{2}$ hours of spontaneous respiration with the animal breathing a mixture of air and oxygen, blood gases were still nearly normal $\left(p \mathrm{H} 7 \cdot 3\right.$, standard bicarbonate $25, \mathrm{PCO}_{2}$ 58, $\mathrm{Po}_{2}$ 183).

The animal continued in this satisfactory state, maintaining a constant blood pressure of 150 / $100 \mathrm{~mm}$. $\mathrm{Hg}$, and a venous pressure fluctuating between 4 and $6 \mathrm{~cm}$. $\mathrm{H}_{2} \mathrm{O}$. The return of spontaneous movements and reflexes was observed during this period. Six-and-a-half hours after closure of the chest respiration became laboured and then stopped suddenly, followed by a rapid fall in blood pressure. Conditions were stabilized by mechanically ventilating the dog and administering a small dose of adrenaline. From this time onwards a slow adrenaline infusion was required to maintain an adequate blood pressure, and the animal did not again show any adequate spontaneous respiratory movements.

The animal continued in this state, passing adequate quantities of urine, until its death 25 hours after operation. Death resulted from irreversible ventricular fibrillation, which occurred suddenly and for no obvious reason when the animal was being lifted on to an $x$-ray table.

In this group death has generally resulted from a gradual decline in respiratory performance followed by relatively sudden cardiac failure.

Two animals have breathed with normal rate, depth, and rhythm in the early post-operative phase but later showed progressive inadequacies of oxygenation, which may be related to areas of patchy collapse seen on chest radiographs.

Radiology has also revealed at least one case of acute gastric dilatation, perhaps a result of vagus nerve damage in the chest.

In several dogs we have electrocardiographic evidence of electrical conduction across the atrial suture line at least for a short period after implantation. The E.C.G.s showed two $P$ waves the recipient $\mathbf{P}$ wave first and the donor atriuno contracting subsequently (Fig. 4). It should be remembered that the recipient SA node and righe atrium have lost their main blood supply, and $\underset{\mathscr{D}}{\mathbb{D}}$ subsequently, ischaemic changes lead to cessation of function of the recipient sino-atrial node.

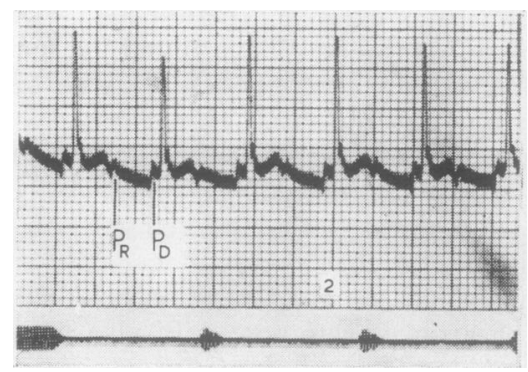

FIG. 4. E.C.G. following cardiopulmonary transplantation showing the relationship between the recipient $P$ wave $(P R)$ and the donor $P$ wave $(P D)$.

\section{DISCUSSION}

Although we have been unable to achieve long $\stackrel{\overrightarrow{0}}{\overrightarrow{0}}$ term survival we have been impressed with the good cardiac function obtained following trans? plantation. Respiratory failure has occurred in $a$. large number of our animals and it appears that most dogs do not tolerate bilateral lung denerva tion. Further work in our laboratory (Sekabunga, Cooper, and Manders, 1969) has confirmed this and concurs with the studies of Webib and his colleagues (Webb and Howard, 1957 ; Howard and Webb, 1957 ; Webb, deGuzman, and Hoopes, 1961 ; Nakae, Webb, Theodorides, and Sugg, $?$ 1967). However, it is likely that a small number of dogs would survive following this procedure. Survivors reported by Demikhov (1962) and N Lower, Stofer, Hurley, and Shumway (1961) which breathed adequately for 6 and 5 days respectively until bronchopneumonia or rejection $\omega$ caused death, give weight to the possibility that a few dogs can tolerate total denervation of botho lungs.

It seems that afferent impulses originating in ${ }^{\text {? }}$ the lungs are essential to the maintenance of ${ }_{-}^{\underline{T}}$ respiration in the majority of dogs. Severance of these afferent pathways leads to respiratory failure. It is possible that an alternative pathwayo from afferent receptors in the chest wall and dia-O phragm is able to maintain the sensory pathwayso to the central nervous system in a few dogs aftero 
severance of the normal vagal fibres from the lungs. Possibly the number of afferent receptors present in these sites in individual animals is a determining factor in their length of survival following bilateral pulmonary denervation.

There is evidence of a difference between the primate and the $\operatorname{dog}$ in dependence on the Hering-Breuer reflex. Haglin, Telander, Muzzall, Kiser, and Strobel (1963) obtained good results in baboons surviving on a single reimplanted lung. Nakae and his associates (1967) have also obtained survival with no evidence of respiratory failure in Macacca Rhesus monkeys subjected to total mediastinal dissection and high tracheal transection.

These findings have considerable significance in regard to human cardiopulmonary transplantation. It is likely that the neural control of respiration in man resembles that of the primates, and therefore it is hoped that man will satisfactorily tolerate transplantation of the heart and both lungs.

\section{ETHICAL AND ORGANIZATIONAL CONSIDERATIONS}

As we have tried to show, many of the apparent special problems associated with heart and lung transplantation are likely to be overcome. The ethical considerations which have received so much publicity will be allayed if we can demonstrate in man a method of resuscitating and preserving hearts an hour or more after the death of the donor. As early as 1936 Kountz revived human hearts up to six hours after death in order to study their physiology. The probable shortage and wastage of donor material will be minimized if we have adequate tissue typing services and a large recipient pool of patients already typed. The recipient pool can be increased sufficiently to cover rare tissue groups only if there is a data-handling service and a simple method of transporting tissues.

While transplantation remains a complicated procedure requiring cardiopulmonary bypass, it will be necessary for the operation to be performed in specialist centres. This means that donor material will have to be transported to these centres. We feel that the transportation system must be simple, and suggest that the methods outlined above may be adequate and will be socially acceptable.

Tissue typing in life and realistic legislation about donor material will reduce the burden on the tissue typing organization.

Already such a service exists in the form of a pilot scheme run by Professor J. R. Bachelor at East Grinstead, and other centres are setting up tissue typing facilities.

Since a transplantation is triggered by an emergency in the form of an available donor, efficient organization is required to avoid the necessity of experienced teams remaining on call with vacant theatres, as well as reducing wastage due to tissue mismatch.

If heart transplantation is to turn promise into performance, adequate organization is as imperative as advances in surgical techniques. Van Rood has taken a major step towards this by setting up the Eurotransplant service. Potential recipients are tissue typed and the data are stored in computer-readable form. As tissues become available they, too, are typed and matched with recipients by the computer. The service is in use. On Wednesday, 6 December 1967 , it selected a Leiden woman for a kidney from an accident victim at Deventer on the other side of Holland. Van Rood's system could be extended to include voluntary donors who will be inconspicuously tattooed with their tissue type and blood group, or who will carry a suitable tally. Because of the emergency nature of the service, the Human Tissues Act will have to be revised so that such marks legally constitute proof of consent to the removal of organs after death without reference to next of kin.

Since any such system works by matching demand with sudden availability, the wider the catchment area, the better the total match. Ultimately, then, the national and continental services that come into being will merge to form the global service that supersonic aircraft will make possible within a decade (Longmore, 1968).

The world service would handle information about organs and transport facilities available. The actual tissues would be routed by common carriers such as taxis and aircraft between hospitals.

It will be seen that a simple, inexpensive method of transporting tissues, developed from the methods we have suggested, will be essential for such an organization. Nothing in this organization is at all extraordinary by current technological standards. Indeed, the World Meteorological Organization has already set an excellent precedent in the rapid handling of data through a global network. Without such an organization surgeons will be forced to hunt locally for tissues. Even though they and their colleagues may feel sure that no patient's chances, however small, are jeopardized to help other patients with better prognoses, a certain measure of public scepticism on this point would be understandable. An international service could work efficiently without having any recourse to equivocal cases and, in this way, public confidence in ethical practice would not be lost.

From the facts discussed emerge four requirements :

1. Reassurance of the public;

2. Guidance as to a definition of death which takes into account future advances in resuscitation and life support techniques ;

3. A new Human Tissues Act;

4. An organization for a national tissue service 
which can later be integrated with Eurotransplant and a World Tissue Service.

The solution of these ethical and organizational problems must develop in parallel with advances in surgical techniques and in the control of the immune reaction.

We should like to thank Sir Thomas Holmes Sellors, D.M., M.Ch., F.R.C.S., F.R.C.P., and Professor E. C. Amoroso, F.R.S., F.R.C.S., F.R.C.V.S., for their guidance and help. Major contributions to this work have been made by the following British Heart Foundation Fellows: B. Gersh, M.B., Ch.B. Cape Town; C. S. Nelson, F.R.C.S. ; and R. Sarin, M.B. Punjab., Ch.M. Liverpool, F.R.C.S.E., F.R.C.S., and by many technicians, nurses, and helpers from the National Heart Hospital.

This work was supported by the British Heart Foundation, the Halley Stewart Trust, the National Heart Hospital, and the Institute of Cardiology.

\section{REFERENCES}

Cooper, D. K. C. (1969). Experimental development of cardiac transplantation. Brit. med. J., 4, 174.

- (1969). Transplantation of the heart and both lungs. 1: Historical review. Thorax, 24, 383.

Demikhov, V. P. (1950). A new and simpler variant of heart-lung preparation of a warm-blodded animal. Byull. éksp. Biol. med., no. 7 .
(1962). In Experimental Transplantation of Vital Organs. $\stackrel{\overline{\bar{N}}}{+}$ Authorised translation from the Russian by Basil Haigh. Consultants Bureau, New York.

Haglin, J., Telander, R. L., Muzzall, R. E., Kiser, J. C., and Strobel, C. J. (1963). Comparison of lung autotransplantation in the primate and dog. Surg. Forum, 14, 196.

Hardy, J. D., and Alican, F. (1966). Lung Transplantation, in Advances in Surgery, Vol. 2, p. 235. Ed. Welch, C. E. Year Book Medical Publishers, Chicago.

Howard, H. S., and Webb, W. R. (1957). Respiratory paralysis $-\vec{C}$ following pulmonary denervation. Surg. Forum, 8, 466.

Kountz, W. B. (1936). Revival of human hearts. Ann. intern. Med., $\rightarrow$ 10,330 .

Longmore, D. B. (1968). Implants or transplants? Sci. J., 4, no. 2 హ్ (Feb.), p. 78

Lower, R. R., Stofer, R. C., Hurley, E. J., and Shumway, N. E. (1961). Complete homograft replacement of the heart and both
lungs. Surgery, 50, 842.

Nakae, S., Webb, W. R., Theodorides, T., and Sugg, W. L. (1967). it Respiratory function following cardiopulmonary denervation $\omega$ in dog, cat, and monkey. Surg. Gynec. Obstet., 125, 1285.

Registrar General (1967). Statistical Review of England and Wales for the Year 1965: Part 1. H.M.S.O., London.

Robicsek, F., Sanger, P. W., and Taylor, F. H. (1963). Simple methed of keeping the heart "alive" and functioning outside of the body for prolonged periods. Surgery, 53, 525 .

Sekabunga, J., Cooper, D. K. C., and Manders, J. (1969). Effects of total cardiopulmonary denervation in dogs. In preparaticn.

Shumway, N. E., Lower, R. R., and Stofer, R. C. (1966). Trans-c plantation of the heart, in Advances in Surgery, Vol. 2, p. 265.\% Ed. Welch, C. E., Year Book Medical Publishers, Chica go.

Webb, W. R., and Howard, H. S. (1957). Cardio-pulmonary trarsplantation. Surg. Forum, 8, 313.

- deGuzman, V., and Hoopes, J. E. (1961). Cardiopulmonary transplantation: Experimental study of current problems. Amer. Surgn, 27, 236.

Yamada, T., Bosher, L. H., and Richardson, G. M. (1965). Observations on the autoperfusing heart-lung preparation. Trans. Amer. Soc. artif. intern. Org., 11, 192. 